\title{
Association of sex hormone-binding globulin and free testosterone with mortality in men with type 2 diabetes mellitus
}

\author{
Aye N Tint ${ }^{1}$, Rudolf Hoermann², Henry Wong ${ }^{1}$, Elif I Ekinci ${ }^{1,2}$, Richard J Maclsaac ${ }^{2,3}$, \\ George Jerums ${ }^{1,2}$, Jeffrey D Zajac ${ }^{1,2}$ and Mathis Grossmann ${ }^{1,2}$ \\ ${ }^{1}$ Department of Endocrinology and Diabetes, Austin Health, 145 Studley Road, Heidelberg, Victoria 3084, Australia, \\ ${ }^{2}$ Department of Medicine, University of Melbourne, Victoria, Australia and ${ }^{3}$ Department of Endocrinology and \\ Diabetes, St Vincent's Hospital, Melbourne, Victoria, Australia
}

\author{
Correspondence \\ should be addressed \\ to M Grossmann \\ Email \\ mathisg@unimelb.edu.au
}

\begin{abstract}
Objective: Low circulating testosterone levels have been associated with increased mortality in men. We hypothesized that the prognostic role of testosterone in men with type 2 diabetes mellitus (T2DM) is influenced by its carrier protein sex hormone-binding globulin (SHBG).

Design: We conducted a prospective cohort study at a tertiary referral centre.

Methods: In total, 531 men with T2DM presenting to a diabetes clinic in 2004-2005 were followed prospectively until death, or July 31, 2014, and a survival analysis was performed. The main outcome measure was all cause mortality.

Results: Over a mean (s.D.) follow up of 7.6 years (2.6) 175 men (33\%) died. In Cox proportional hazard models both higher SHBG (Hazard Ratio (HR) $1.012(95 \% \mathrm{Cl} 1.002-1.022), P=0.02)$ and lower calculated free testosterone (cFT) (HR 0.995 (95\% Cl 0.993-0.998), $P=0.001$ ) were risk factors for all cause mortality independently of age, BMI, presence of macro- and microvascular disease, duration of T2DM, hemoglobin, renal function, insulin use, C-reactive protein and homeostatic model of insulin resistance. By contrast, the inverse association of total testosterone (TT) with mortality weakened after these adjustments $(P=0.11)$. SHBG remained associated with mortality $(P<0.001)$ both if substituted for or added to TT in the multivariable model. In the fully adjusted model, an increase of SHBG by $17.3 \mathrm{nmol} / \mathrm{l}$ (1 s.D.) increased mortality by $22 \%$ and a decrease in CFT by $81 \mathrm{pmol} / \mathrm{l}$ (1 s.D.) increased mortality by $45 \%$.

Conclusions: The association of SHBG with mortality in men with T2DM is novel. Whether SHBG acts via regulation of testosterone, has intrinsic biological roles, or is a marker of poor health requires further study.
\end{abstract}

\section{Introduction}

Sex hormone binding globulin (SHBG) has traditionally been considered to be a passive carrier for circulating sex steroids such as testosterone, determining their bioavailability and metabolic clearance (1). In men, $40 \%$ of serum testosterone is tightly bound to SHBG, 58\% loosely to albumin, and the remaining $0.5-2 \%$ circulates as the free form. More recent evidence suggests that SHBG is not just a passive carrier, but actively regulates the cellular uptake of testosterone and cellular proliferation via a specific receptor $(1,2)$. SHBG gene polymorphisms influence the risk of developing type 2 diabetes mellitus (T2DM) (3), and SHBG has been implicated in the development of the metabolic syndrome independent of testosterone levels (4). Circulating SHBG concentrations are strongly correlated to testosterone levels and affected by a variety of factors. Most importantly, SHBG levels increase with age, whereas SHBG levels are reduced with obesity or insulin resistance (5). While low endogenous testosterone levels have been associated with increased mortality in observational studies of community-dwelling men (6), only one

Published by Bioscientifica Ltd 
study has assessed this association specifically in men with T2DM (7), with inconclusive findings. Focus on men with T2DM is important not only because they commonly have reduced testosterone levels (8) but also because total testosterone (TT) levels, generally accepted to be the most useful diagnostic measures of androgen status may be confounded by low SHBG levels due to insulin resistance and obesity (5). Moreover, prognostic implications of SHBG in men with T2DM have not been investigated previously.

To assess whether SHBG and testosterone are risk factors for mortality independent of competing mortality factors or factors that influence their circulating concentrations, we prospectively followed a well-characterized cohort of men with T2DM who had a detailed baseline assessment between 2004 and 2005 (9). We hypothesized that if SHBG influences mortality via regulation of testosterone, SHBG will predict mortality additively to total, but not to free testosterone.

\section{Subjects and methods}

\section{Study population}

This study was initiated in February 2004 as a prospective cohort study of patients with T2DM presenting to an outpatient diabetes clinic at Austin Health, Melbourne, Australia, a tertiary referral center. Collection of baseline clinical, anthropometric and laboratory data was performed during routine clinical visits for patients attending between February 2004 and December 2005 as described and in part reported previously (9). SHBG and testosterone levels were measured at baseline and during subsequent clinic visits in blood samples drawn in the morning in the fasted state. Patients were followed to death or to July 31, 2014. All men gave informed written consent and the study was approved by the Austin Health Human Research Ethics Committee.

\section{Ascertainment of incident deaths}

The primary outcome was death from any cause until July 31, 2014. Timing and causes of death were ascertained from the National Death Index (NDI) of the Australian Institute of Health and Welfare (AIHW). This registry contains both the original death certificate and the International Classification of Disease 10th revision (ICD-10) coded cause of death. At the time of linkage all deaths occurring up to July 31, 2014 had been recorded in the NDI. Due to the delay in the ICD-10 coding for cause of death by the AIHW, cause-specific mortality was available up to October 31, 2013.

\section{Quantification of testosterone and SHBG levels}

SHBG levels were determined with the Immulite 2000 analyser (Diagnostics Products Corporation, Los Angeles, CA, USA). Reference range for SHBG was $13-71 \mathrm{nmol} / \mathrm{l}$, and minimum detection limit was $0.02 \mathrm{nmol} / \mathrm{l}$. Intra- and interassay coefficient of variation (CVs) for two different concentrations ( 5.0 and $75.9 \mathrm{nmol} / \mathrm{l}$ ) were $4.2 \%, 5.2 \%$ and $4.6 \%, 5.4 \%$ respectively (9).

Serum TT was measured by immunoassay (Beckman Coulter, Inc., Fullerton, CA, USA) with a minimum detection limit of $0.35 \mathrm{nmol} / \mathrm{l}$ as described. Intra- and interassay CVs assessed for two different concentrations (4.7 and $26 \mathrm{nmol} / \mathrm{l}$ ) were $3.9 \%, 4.8 \%$ and $5.7 \%, 5.0 \%$ respectively. Reference range for this TT assay was $10-27.6 \mathrm{nmol} / \mathrm{l}$, based on gas chromatography/mass spectrometry measurements obtained from a reference panel of 124 healthy, reproductively normal young men $(9,10)$.

Free testosterone values were calculated from TT, SHBG and serum albumin based on mass action laws with Vermeulen's formula (11). Reference range for the AccessTestosterone/Immulite SHBG combination for calculated free testosterone (cFT) was $230-610 \mathrm{pmol} / \mathrm{l}$, based on the same reference panel of 124 healthy, reproductively normal young men $(9,10)$.

\section{Statistical analysis}

The mean and s.D. are reported for descriptive data. Between-group differences were tested for significance by means of Welch $t$-test and $\chi^{2}$-test with Yates' correction for continuity in case of frequency distributions. Only for highly non-normally distributed values such as CRP we used median, interquartile range and Wilcoxon rank sum test. Tables were considered explanatory and not corrected for multiple testing. Correlation between sex hormones was assessed using Pearson's product-moment correlation. Reliability of single hormone measurements was assessed with the use of a mixed random-effects model, including the test variable measured at all time points, the yearly visits as a grouping factor and a subject-related random effect. We estimated the intra-class correlation coefficient (ICC 2) across all time points from patients who had repeated hormone measurements available weighted for sample size at each time point.

Survival analysis was used to follow up patients until they died or their observation period ended. Patients had 
entered the study at various time points between 2004 and 2005 , but the study period ended for all patients on July 31,2014 , the date of the mortality audit. The status of all patients was confirmed on this date. As a result, the uncensored observation period extended to a minimum of 8.6 years where every subject was accounted for, but was variable thereafter.

Analyses of risk factors associated with increased mortality relied on Cox Proportional Hazards model. Differences in frequencies were compared by log rank test, significance of covariates in multivariable models based on Wald test. The assumption of proportional hazards was verified by Schoenfeld test and Schoenfeld plots. Analyses were carried out with the covariates as continuous or categorical variables. Normally, the effect of a continuous covariate on the hazard is assumed to have a log-linear functional form in the Cox Proportional Hazards model. We tested for linearity of a continuous covariate in the Cox Proportional Hazard model by fitting penalised smoothing splines of three degrees with the default four degrees of freedom (psplines in the R package). A nonlinear functional form for a covariate was retained in the model only if the nonlinear component of the fit was confirmed to be significant by Wald test. This was the case for univariable models with TT and cFT, whereas a linear approximation was sufficient for the multivariable models involving the two androgens. BMI showed a u-shaped relationship and was therefore discretised into meaningful clinical categories for use as a covariate in the models. For SHBG, age and other continuous covariates, the assumption of a log-linear hazard was not violated either in the uni- or multivariable models. In the models reported, results refer to the use of the linear continuous variable unless stated otherwise. Despite the proportionality assumption not being violated, we tested linearity of age by fitting splines to age, stratified for age using the built-in 'strata' routine of the program, and assessed its interaction with androgens. In the final model, we relied on age as a linear continuous covariate, as none of the more complex functions provided significantly better estimates. For risk factors of interest, such as SHBG, cFT and TT, crude and adjusted hazards ratios are reported to ascertain their significance independently of covariates. Candidate covariates for hazard models were chosen a priori to cover covariates affecting competing diabetes-related mortality risk markers, including i) in multivariable model 1: age and four categories of BMI $(<25,25-30,30-35$, $\left.>35 \mathrm{~kg} / \mathrm{m}^{2}\right)$, ii) in multivariable model 2 , in addition to the factors present in model 1: duration of T2DM, presence of macro- and microvascular disease and iii) in multivariable model 3: all factors of model 2 and C-reactive protein (CRP), haemoglobin, estimated glomerular filtration rate (eGFR), insulin use and the homeostatic model of insulin resistance (HOMA-IR) as a measure of insulin resistance. Surrogate markers such as $\mathrm{HbA1c}$ were not included in the final model because i) $\mathrm{HbA1c}$ was not a significant risk factor in a univariable model in this cohort $(P=0.33)$; ii) we already included a panel of more robust end point markers such as duration of diabetes, presence of macro and microvascular complications and insulin resistance; and iii) concerns of over-fitting due to collinearity with the other markers. We did, however, test that the inclusion of HbA1c did not weaken the association of SHBG and androgens in multivariable model 1 (see the section 'Results'). In addition, metformin use was not included in the model, because metformin use was related to age, renal function and prognostic markers of diabetes as well as to SHBG and testosterone levels, making incorporations of metformin use highly complex.

Cause-specific hazards models were censored for competing risks. The significance of an added factor on improving model prediction was assessed in nested models with the likelihood ratio test. The performance of the various predictive models was compared with receiver operating characteristics (ROC) curves derived by generalized linear models with a binomial function (binary logistic regression) using survival status at 8.6 years as the outcome. ROC curves were tested for significant difference with Delong's test. $P<0.05$ conferred significance throughout. The statistical software packages R 3.2 for Mac, JGR 1.7-17, Deducer 0.7-7, nlme 3.1-120, packHV 1.8, rms 4.3-1 and pROC 1.8 were used for the data analyses $(12,13)$.

\section{Results}

\section{Baseline characteristics of the patients}

Of the original cohort of 571 patients with T2DM (9), 40 men were excluded because they were subsequently commenced on testosterone treatment $(n=25)$, or received androgen deprivation therapy for prostate cancer $(n=15)$, leaving an analytical sample of 531 men. The baseline characteristics of the 531 men are shown in Table 1. Mean age (s.D.) was 66 years (11.7), BMI $30.1 \mathrm{~kg} / \mathrm{m}^{2}$ (5.3), SHBG $37 \mathrm{nmol} / \mathrm{l}$ (17.3), TT $11.2 \mathrm{nmol} / \mathrm{l}$ (4.3) and cFT $223 \mathrm{pmol} / \mathrm{l}(81)$.

During the observation time (mean 7.6 years, s.D. 2.6), 175 men (33\%) died. Mean follow up of survivors was 9.1 years (0.4), and mean time to death was 4.6 years (2.6). 
Table 1 Baseline characteristics of the patients. Values show mean and (s.D.) or number (\% of total) for each parameter, except for CRP, where values show median and interquartile (25th to 75th percentile) range. Men who died had entered the study slightly earlier than those who were still alive at the end of the observation period, resulting in a slightly shorter theoretical observation time: mean (s.D.) 9.1 (0.4) for men who remained alive compared to 9.2 (0.4) for men who died, $P=0.003$.

\begin{tabular}{|c|c|c|}
\hline Parameter & All $(n=531)$ & Alive $(n=356)$ \\
\hline Age (years) & $66(11.7)$ & $62(11.0)$ \\
\hline BMI $\left(\mathrm{kg} / \mathrm{m}^{2}\right)$ & $30.1(5.3)$ & $30.1(4.9)$ \\
\hline SHBG (nmol/l) & 37 (17.3) & $34(16.0)$ \\
\hline TT (nmol/l) & $11.2(4.3)$ & $11.7(4.2)$ \\
\hline cFT (pmol/l) & $223(81)$ & $241(75)$ \\
\hline HbA1c (\%) & $7.5(1.3)$ & $7.5(1.2)$ \\
\hline HOMA-IR & $2.58(1.6)$ & $2.48(1.5)$ \\
\hline Cholesterol (mmol/l) & $4.3(0.9)$ & $4.4(0.8)$ \\
\hline Triglycerides (mmol/l) & $1.7(1.2)$ & $1.7(1.2)$ \\
\hline $\mathrm{HDL}(\mathrm{mmol} / \mathrm{l})$ & $1.3(0.4)$ & $1.3(0.4)$ \\
\hline Haemoglobin (g/l) & $142(16.0)$ & $146(14.2)$ \\
\hline Albumin $(g / l)$ & 40 (3.6) & $40(3.0)$ \\
\hline eGFR (ml/min) & $71(24.7)$ & $76(22.9)$ \\
\hline CRP (nmol/l) & $3.2(1.1-6.2)$ & $2.4(0.9-5.5)$ \\
\hline \multicolumn{3}{|l|}{ Insulin use } \\
\hline No & $294(58 \%)$ & $208(61 \%)$ \\
\hline Yes & $215(42 \%)$ & $131(39 \%)$ \\
\hline \multicolumn{3}{|l|}{ Macrovascular disease } \\
\hline No & $291(57 \%)$ & $224(66 \%)$ \\
\hline Yes & $218(43 \%)$ & $114(34 \%)$ \\
\hline \multicolumn{3}{|l|}{ Microvascular disease } \\
\hline No & $276(54 \%)$ & $197(59 \%)$ \\
\hline Yes & $231(46 \%)$ & $139(41 \%)$ \\
\hline Duration of diabetes (years) & $10(5.0-15)$ & $9(5-13)$ \\
\hline
\end{tabular}

\begin{tabular}{c}
\hline Died $(n=175)$ \\
\hline $73(9.6)$ \\
$30.2(6.0)$ \\
$43(18.6)$ \\
$10.2(4.4)$ \\
$185(79)$ \\
$7.4(1.4)$ \\
$2.79(1.8)$ \\
$4.2(1.0)$ \\
$1.7(1.1)$ \\
$1.3(0.4)$ \\
$135(17.0)$ \\
$39(4.4)$ \\
$59(25)$ \\
$3.9(2.2-7.4)$ \\
$86(51 \%)$ \\
$84(49 \%)$ \\
$67(39 \%)$ \\
$104(61 \%)$ \\
$79(46 \%)$ \\
$92(54 \%)$ \\
$13(7-19)$ \\
\end{tabular}

\begin{tabular}{cc}
\hline P value \\
\hline$<0.001$ \\
0.88 \\
$<0.001$ \\
$<0.001$ \\
$<0.001$ \\
0.33 \\
0.072 \\
0.098 \\
0.87 \\
0.85 \\
$<0.001$ \\
0.006 \\
$<0.001$ \\
$<0.001$ \\
0.026 \\
\\
$<0.001$ \\
\\
\end{tabular}

\begin{tabular}{c}
\hline $\boldsymbol{N}$ \\
\hline 531 \\
498 \\
531 \\
531 \\
531 \\
531
\end{tabular}

502

450

449

449

449

531

448

448

509

$86(51 \%)$

$67(39 \%)$

509

Compared to men who remained alive, men who died

When further analyzing hazards by Cox proportional during follow-up were older (73 years (9.6) vs 62 years (11.0), $P<0.001$ ), had longer duration of T2DM, and a higher prevalence of established macro- and microvascular disease. They also had worse renal function, lower haemoglobin levels and higher CRP levels at initial presentation (Table 1). There was no difference in BMI, HbA1c, lipid levels or HOMA-IR between the men who died or remained alive (Table 1 ).

\section{Associations of baseline sex steroid levels with all-cause mortality}

Men who died during follow-up had higher baseline SHBG levels compared to the men who remained alive: mean (s.D.) $43 \mathrm{nmol} / 1$ (18.6) vs $34 \mathrm{nmol} / 1$ (16.0), $P<0.001$ (Table 1). In contrast, baseline testosterone was lower in the men who died compared to men who remained alive: TT $10.2 \mathrm{nmol} / \mathrm{l}$ (4.4) vs $11.7 \mathrm{nmol} / \mathrm{l}$ (4.2), $P<0.001$ and cFT $185 \mathrm{pmol} / \mathrm{l}$ (79) vs $241 \mathrm{pmol} / \mathrm{l}(75), P<0.001$.

Survival was significantly worse in men with higher baseline SHBG and with lower testosterone levels, as shown by Kaplan-Meier plots (Fig. 1A, B and C). hazard models, in univariable analyses, SHBG was associated positively $(P<0.001)$ and TT $(P<0.001)$ and $\mathrm{cFT}$ $(P<0.001)$ inversely with mortality. It should be noted that, while no violations of the proportional hazard assumption occurred, for TT a nonlinear penalised spline curve fitted the log hazard significantly better $(P=0.007$ for the non-linear component) than a linear approximation (Table 2). This indicates a threshold effect for TT starting at approximately $10 \mathrm{nmol} / 1$. A linear approximation tends to slightly underestimate the risk of low TT levels. For example, a TT level of $5 \mathrm{nmol} / \mathrm{l}$ confers an estimated relative hazard rate of 2.1 (95\% CI 1.6-2.7) in the nonlinear model, compared to 1.7 (95\% CI 1.3-2.1) in a linear model. Results for TT with age strata (HR 0.95 (95\% CI 0.91-0.99)) were similar to introducing age as a continuous covariate (HR 0.95 (95\% CI 0.92-0.99)). Age did not significantly $(P=0.43)$ interact with TT. Nor was a spline fit of age superior to linear approximation. For SHBG, unlike in TT, the contribution of the non-linear component was not significant $(P=0.52)$. However, a significant $(P=0.014)$ non-proportional influence of age on SHBG was apparent when testing for their interaction 


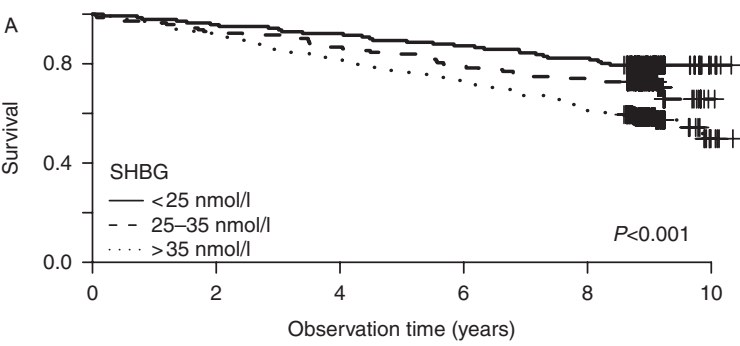

$\begin{array}{lllllll}\text { \# at risk } & & & & & \\ <25 \mathrm{nmol} / \mathrm{l} & 141 & 135 & 130 & 123 & 116 & 5 \\ 25-35 \mathrm{nmol} / \mathrm{l} & 143 & 133 & 124 & 113 & 105 & 1 \\ >35 \mathrm{nmol} / \mathrm{l} & 247 & 225 & 202 & 181 & 151 & 5\end{array}$

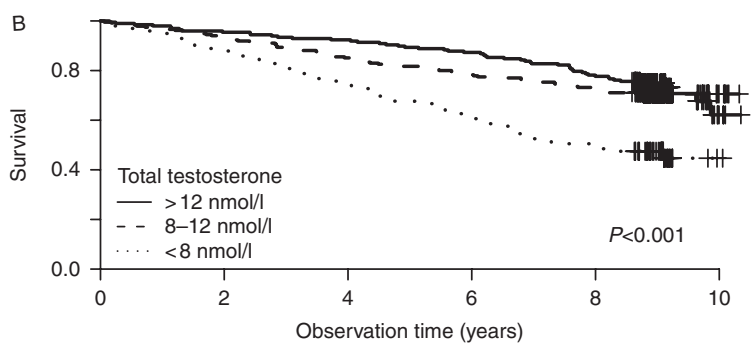

$\begin{array}{lcccccc}>12 \mathrm{nmol} / \mathrm{l} & 197 & 188 & 182 & 172 & 153 & 4 \\ 8-12 \mathrm{nmol} / \mathrm{l} & 235 & 217 & 200 & 184 & 170 & 6 \\ <8 \mathrm{nmol} / / & 99 & 88 & 74 & 61 & 49 & 1\end{array}$

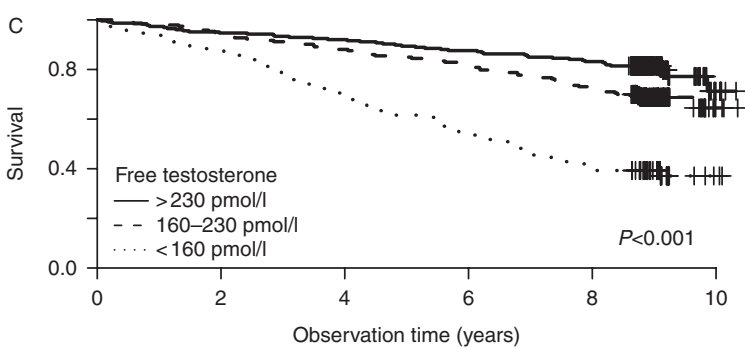

\# at risk

$>230 \mathrm{pmol} / \mathrm{l} \quad 226$ 160-230 pmol// 193 $<160 \mathrm{pmol} / \mathrm{l} \quad 112$

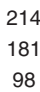

\section{8}

198

158

61

$\begin{array}{ll}188 & 6 \\ 139 & 3 \\ 45 & 2\end{array}$

\section{Figure 1}

Kaplan-Meier estimates of overall survival of patients according to serum SHBG (Fig. 1A), total testosterone (Fig. 1B) and calculated free testosterone (Fig. 1C) levels.

(SHBG, HR 1.090 (95\% CI 1.025-1.158), age, HR 1.128 (95\% CI 1.084-1.174), SHBG*age, HR 0.999 (95\% CI 0.998-0.999)). Younger men showed a more pronounced increase in mortality with increasing SHBG than older men. cFT displayed both a nonlinear log hazard in the univariable model $(P=0.029)$ and a significant $(P=0.040)$ interaction with age, making it a complex risk factor. Its disproportional influence appeared to be stronger in younger men. The influence of these interactions diminished in the more complete models. Thus, we relied on linear approximations with SHBG and the androgens as continuous variables to derive the HRs for the multivariable models reported in Table 2 , where the contributions of the more complex nonlinear components were no longer significant.

Both SHBG and testosterone retained a strong and independent role in various multivariable Cox models adjusting for other known predictors or candidate confounders (Table 2). In particular, SHBG was a risk factor for mortality independently of age, BMI, duration of T2DM, presence of macrovascular and microvascular disease, haemoglobin, eGFR, insulin use, CRP and HOMA-IR $(P=0.017)$ (Table 2$)$. Using Pearson's productmoment correlation, the correlation between TT and SHBG was $0.44, P<0.001$. SHBG remained predictive both if substituted for $(P<0.017$ vs $P=0.11$ for TT) or added to TT levels (likelihood-ratio test, $P<0.001$ ) in multivariable model 3 , both suggesting that SHBG is a stronger predictor of mortality relative to TT. Conversely, adding TT to SHBG improved prediction (likelihood-ratio test, $P<0.001)$ suggesting that TT provides prognostic information not captured by SHBG alone.

Lower TT was a risk factor for mortality independently of age, BMI, duration of T2DM, presence of macrovascular or microvascular disease $(P=0.017)$, but this inverse association was no longer significant when additional adjustments for haemoglobin, eGFR, insulin use, CRP and HOMA-IR were performed $(P=0.11)$ (Table 2$)$. HbA1c was not part of the models for reasons discussed in Methods. However, it did not weaken the association of SHBG $(P=0.026)$, cFT $(<0.001)$ or TT $(P=0.030)$ in multivariable model 1.

A lower cFT was a risk factor for mortality independently of age, BMI, duration of T2DM, presence of macrovascular or microvascular disease, haemoglobin, eGFR, insulin use, CRP or HOMA-IR $(P=0.001)$ (Table 2 ). Further, when SHBG was added to the fully adjusted model containing cFT, model prediction did not improve (likelihood-ratio test, $P=0.11$ ) whereas conversely adding cFT to the model containing SHBG improved prediction (likelihood-ratio test, $P=0.004$ ). This suggests that a low cFT is a stronger predictor of mortality relative to SHBG.

In the fully adjusted model, an increase of SHBG by $17.3 \mathrm{nmol} / \mathrm{l}$ (1 s.D.) increased mortality by $22 \%$ and a decrease in cFT by 81 pmol/1 (1 s.D.) increased mortality by $48 \%$.

In addition to time-related mortality risks analysed by Cox models, we further investigated the performance of the various predictors by logistic regression using survival status at 8.6 years as a binary outcome. ROC curves derived 
Table 2 Associations of SHBG and testosterone with all-cause mortality. Multivariable model 1: adjusted for age and BMI. Multivariable model 2: adjusted for parameters in multivariable model 1, as well as duration of T2D, presence of macrovascular and microvascular disease. Multivariable model 3: adjusted for parameters in multivariable model 2, as well as hemoglobin, CRP, eGFR, insulin use and HOMA-IR. Sample size $(n)$ and number of events (e) for each model: univariable model $n=531, e=175 ;$ multivariable model $1 n=498, e=166$; multivariable model $2 n=487$, e=164; multivariable model $3 n=398, e=122$. Data are reported as hazard ratios and $(95 \% \mathrm{Cl})$ per unit change.

\begin{tabular}{|c|c|}
\hline Parameters & Unadjusted univariable model \\
\hline SHBG & $\begin{array}{c}1.021(1.013-1.029) \\
P<0.001\end{array}$ \\
\hline TT & $\begin{array}{c}0.921(0.886-0.958) \\
P<0.001\end{array}$ \\
\hline cFT & $\begin{array}{c}0.992(0.990-0.994) \\
P<0.001\end{array}$ \\
\hline
\end{tabular}

\begin{tabular}{c}
\hline Multivariable model 1 \\
\hline $1.010(1.001-1.019)$ \\
$P=0.028$ \\
$0.957(0.920-0.995)$ \\
$P=0.026$ \\
$0.996(0.994-0.998)$ \\
$P<0.001$ \\
\hline
\end{tabular}

\begin{tabular}{c}
\hline Multivariable model 2 \\
\hline $1.010(1.001-1.019)$ \\
$P=0.039$ \\
$0.953(0.915-0.991)$ \\
$P=0.017$ \\
$0.996(0.994-0.998)$ \\
$P<0.001$ \\
\hline
\end{tabular}

\begin{tabular}{c}
\hline Multivariable model 3 \\
\hline $1.012(1.002-1.022)$ \\
$P=0.017$ \\
$0.960(0.912-1.009)$ \\
$P=0.11$ \\
$0.995(0.993-0.998)$ \\
$P=0.001$
\end{tabular}

by the models and compared by the area under the curve (AUC) showed the combination of TT plus SHBG to significantly improve prediction over the use of TT alone (Fig. 2A). Sensitivity and specificity of the predictions are also shown for the more complete models (Fig. 2B). In a linear regression with cFT as the dependent variable and TT and SHBG as the explanatory variables, the adjusted $\mathrm{R}$ squared value was $0.92, P<0.001$. Pearson's productmoment correlations between cFT and SHBG were -0.25 $(P<0.001)$ and, between cFT and TT, $0.72(P<0.001)$.

Sensitivity analyses - Findings did not materially change if deaths occurring within the first 12 months of the observation period $(n=16)$ were excluded, and both SHBG $(P=0.038)$ and cFT $(P<0.001)$ were still associated with mortality after adjustment for all confounders (multivariable Model 3). As in the full-time model, the predictive value of low TT failed to reach significance $(P=0.089)$. In an incomplete sample $(n=198)$, smoking status was not a significant confounder either by itself or in an adjusted model 1 and was therefore not included as a variable in models of the entire population.

\section{Associations of baseline SHBG and testosterone levels with cause-specific mortality}

Information on cause-specific mortality was available for a total of 157 deaths: 49 men (31\%) died of cancer, 46 (29\%) of cardiovascular causes and 62 (39\%) due to other causes.

\section{Cancer mortality}

Both higher SHBG and lower cFT were risk factors for cancer mortality independently of age and BMI; SHBG, HR (95\% CI) 1.027 (1.012-1.043), $P<0.001$ and cFT HR (95\% CI) 0.992 (0.988-0.996), $P<0.001$, but not TT, HR (95\% CI) 0.948
(0.878-1.024), $P=0.18)$. Additional covariates were not examined due to an insufficient number of events. High SHBG remained predictive if additionally adjusted for TT levels in the above model (HR 1.040 (95\% CI 1.022-1.057), $P<0.001)$. Findings for SHBG $(P=0.002)$ and cFT $(P=0.002)$ remained significant after additional exclusion of eight men either diagnosed with cancer diagnosed at baseline $(n=6)$ or dying of cancer deaths in the first 12 months $(n=2)$. Cancer mortality was diverse, with lung (20\%), colorectal (20\%) and hematological (20\%) the most common causes.

\section{Mortality due to cardiovascular disease}

SHBG was not significantly associated with cardiovascular disease (CVD)-related mortality (HR 1.015 (95\% CI 0.9991.031), $P=0.052$ ). Lower cFT was associated with CVD mortality (HR 0.993 (95\% CI 0.989-0.997), $P<0.001$ ), but this was no longer significant after adjusting for age and BMI $(P=0.12)$. TT was not $(P=0.11)$ associated with CVD mortality.

\section{Mortality from causes other than cancer or CVD}

In univariable analyses, SHBG (HR 1.014 (95\% CI 1.001-1.028), $P=0.042$ ), lower cFT (HR 0.993 (95\% CI $0.989-0.997), P<0.001)$ and lower TT (HR $0.899(95 \%$ CI 0.840-0.960), $P=0.002$ ) were associated with increased non-cancer/CVD mortality but these associations became non-significant after adjustment for age and BMI $(P=0.57$, $P=0.25$ and $P=0.073$ respectively).

Stability of hormone levels on repeated measurements $>$ Of the 531 men, 443 men representing for follow-up had one or more repeat hormone levels available, measured after a median of 2.0 years (range $0.6-4.1$ years). 

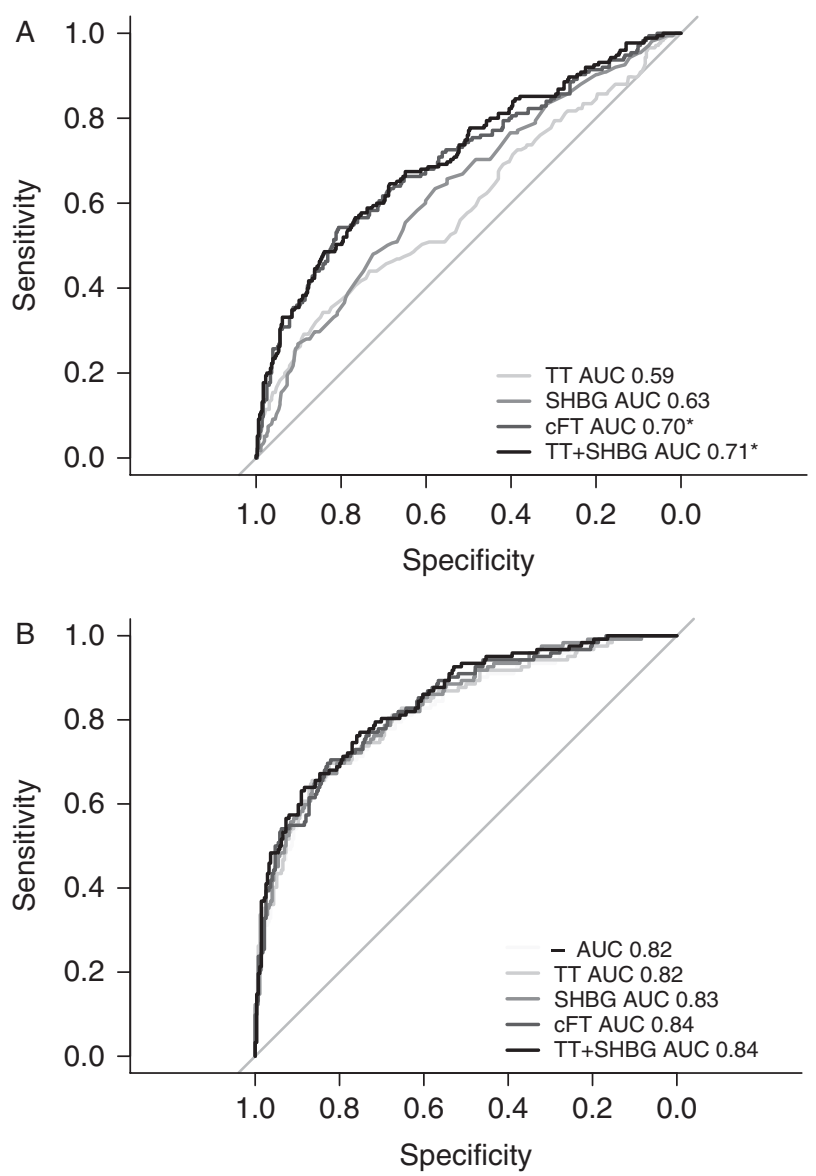

Figure 2

Receiver Operating Curves. (A) Involving each parameter by itself and the combination of SHBG and TT. Asterisk denotes a significant $(P<0.001)$ difference to the model involving TT only. (B) Involving parameters as indicated by the legend in addition to all variables of model 3 .

Baseline characteristics of these men were not signficantly different in men with repeated compared to single hormone measurements except for duration of T2DM, which was shorter (median 8.5 vs 10 years, $P=0.037$ ), and HOMA (2.8 vs $2.2, P=0.032$ ), which was higher in the men with single measurements. In men who had repeated hormone levels, there was a high intraclass correlation between all samples taken, 0.95 for SHBG, 0.93 for cFT and 0.81 for TT.

\section{Discussion}

In this prospective cohort study of men with T2DM, high SHBG and low free testosterone levels were strongly associated with all-cause mortality independently of many recognized diabetes-related risk factors, making them relevant risk factors for long-term mortality. In contrast, the association of low TT levels with mortality weakened after adjustment for confounders.

Given the observational nature of this study, we cannot determine whether these associations are causal, due to reverse causality, or non-causal and driven by shared risk factors. Remarkably, the associations of SHBG and free testosterone remained strongly significant after adjustment for multiple competing mortality risk factors and for patient characteristics affecting the circulating levels of these hormones, including age, BMI, vascular disease, duration of T2DM, inflammation, insulin use, insulin resistance, renal function and haemoglobin. While the association with cancer mortality was strongest, both SHBG and free testosterone were weakly associated with death from cardiovascular and other causes. It is possible that SHBG and free testosterone represent markers of poor health. Consistent with this possibility, malnutrition and energy deficits can increase SHBG levels (14), and acute or chronic illness can reduce circulating testosterone (15). However, the mortality associations persisted after deaths occurring in the first 12 months were excluded. SHBG and free testosterone were associated with mortality outcomes over many years with stable serum concentrations on repeat measurements. This makes it less likely that their levels were affected by unmeasured illness.

While low testosterone has been associated with increased mortality in most but not all studies (6), the prognostic significance of SHBG has received little attention. Assuming high SHBG is causally related to increased mortality, what are the possible mechanisms?

On the one hand, SHBG could affect mortality indirectly, via regulating testosterone action, either via its binding properties or via a receptor-mediated process. Consistent with the former is the free-hormone hypothesis, which postulates that only free testosterone is bioactive whereas SHBG-bound testosterone is not. In this scenario, higher SHBG levels, by binding more testosterone, would limit the amount of bioactive testosterone leading to a reduction in tissue androgenisation. In line with this, low free testosterone was associated with mortality not only more strongly than SHBG but also more strongly than TT. Alternatively, or additionally, SHBG may modulate the activity of bound testosterone via receptor-mediated endocytosis. In rodents, megalin, a member of the low-density lipoprotein receptor gene family, facilitates the endocytosis of SHBG-bound sex steroids into cells, suggesting that SHBG actively regulates testosterone action even in the bound state (2). 
On the other hand, the present findings do not exclude the possibility that SHBG could affect mortality directly, in addition to its role in testosterone regulation. Although not yet cloned, there is strong evidence for the existence of a $G$ protein-coupled SHBG receptor, and SHBG has been shown to regulate cell proliferation in preclinical models (16). Only unliganded, but not sex steroid-bound SHBG binds to the SHBG receptor, suggesting that SHBG may have biological actions that are independent of sex steroids $(1,17)$.

Given that low SHBG levels are associated with the development of T2DM or the metabolic syndrome $(3,4)$, one may expect low SHBG levels to be a risk factor for cardiovascular mortality. In one study of communitydwelling elderly men, low SHBG was associated with increased cardiovascular mortality, but the association was not significant after adjustment for prevalent CVD (18). In addition, SHBG was not associated with mortality in other prospective studies after multivariable adjustments $(19,20)$. Consistent with our findings, high SHBG was independently associated with both all-cause and non-CVD mortality in a large cohort of elderly men (21), and allcause mortality in a small study of men with congestive cardiac failure (22). However, in Norwegian men, a single nucleotide polymorphism (SNP) in the $S H B G$ gene, rs1799941, was not associated with mortality, despite its association with $24.7 \%$ higher SHBG and $14.7 \%$ higher testosterone levels (23). None of these previous studies specifically enrolled a diabetic population. It is plausible that SHBG, given its relationship with insulin resistance, has different prognostic roles in men without diabetes compared to men with established diabetes, with a median duration of T2DM of 10 years in our population.

There has only been one previous study in a cohort of men with T2DM to compare our findings with (7). That study was limited by shorter follow-up of 5.8 years and a lower number of deaths $(n=72)$, and an association of low testosterone with mortality was lost after adjustment for age and SHBG (7).

In our diabetic population, high SHBG and low free testosterone were associated with cancer mortality independent of age and BMI, but not with mortality from other causes. While low testosterone has been associated with cancer mortality in community-dwelling men (24), we are unaware of studies reporting an association of SHBG with cancer mortality, although the aforementioned SNP in the $S H B G$ gene (23) was not associated with incident cancer. Further studies in larger population are required to confirm our observations.
Strengths of this study include a relatively long followup and a large number of prospectively and robustly adjudicated deaths with consistent findings after adjustments for multiple confounding variables and after sensitivity analyses. In addition, given the potential variability of sex steroids levels (5), we performed repeated measurements of testosterone and SHBG and showed that these levels remained stable over time, with intra-class correlation coefficients ranging from 0.81 to 0.93 .

Limitations of this study include the observational design that cannot distinguish causation from association because of possible reverse causation and confounding by unmeasured variables. We studied a high-risk population with relatively longstanding T2DM attending a tertiary referral centre, and whether the findings are generalizable to lower-risk men is unknown. We did not have sufficient information regarding thyroid function or alcohol consumption, variables that may affect SHBG levels (5). Only few patients used anti-convulsive medications or were diagnosed with liver cirrhosis, both linked to increased SHBG levels (5). Indeed, while these conditions usually markedly elevate SHBG levels, in the entire cohort, SHBG levels exceeded the upper limit of normal range by $30 \%$ in only three men. Moreover, only five deaths were due to liver disease. While SHBG immunoassays are robust (25), we measured testosterone levels by immunoassay, rather than by mass spectrometry. However, in a previous RCT of men with T2DM and lowered testosterone levels (26), we observed, prior to testosterone treatment, a high correlation ( $r=0.78, P<0.0001)$ of the testosterone immunoassay used here with a Centers of Disease Control-traceable mass spectrometry assay (27). Although we collected detailed information at baseline, we do not have followup information on whether there was a change in competing risk factors over time. While free testosterone was calculated rather than measured directly, mass-action equations like Vermeulen's formula used here (11) correlate very well with gold-standard equilibrium dialysis (28). Finally, given that only a small number of men $(n=25)$ were commenced on testosterone treatment during follow-up, we cannot assess whether testosterone treatment is associated with changes in mortality.

In summary, this study shows that higher SHBG and lower free testosterone levels are remarkably robust risk factors for mortality in men with diabetes, independently of a large number of competing risk factors or modifiers of circulating hormone concentrations. The fact that only low free but not TT was associated with mortality, coupled with the observation that free testosterone had a stronger impact on mortality than SHBG, suggests that high SHBG 
may affect mortality predominantly by restraining testosterone activity. However, there is no consistent evidence that testosterone treatment improves mortality in men (29), and our findings do not exclude an independent role of SHBG. Therefore the prognostic roles of SHBG and its intrinsic biological actions require further study.

\section{Declaration of interest}

The authors declare that there is no conflict of interest that could be perceived as prejudicing the impartiality of the research reported.

\section{Funding}

M Grossmann was supported by a National Health and Medical Research Council of Australia Career Development Fellowship (\# 1024139).

\section{Author contribution statement}

A $\mathrm{N}$ Tint researched data and wrote the manuscript, $\mathrm{R}$ Hoermann analysed data, contributed to the discussion and reviewed/edited the manuscript. $\mathrm{H}$ Wong researched data and reviewed/edited the manuscript. E I Ekinci, R J Maclsaac, G Jerums and J D Zajac contributed to the discussion and reviewed/edited the manuscript. $M$ Grossmann researched data and wrote the manuscript. A N Tint and M Grossmann are the guarantors for the study.

\section{References}

1 Rosner W, Hryb DJ, Kahn SM, Nakhla AM \& Romas NA. Interactions of sex hormone-binding globulin with target cells. Molecular and Cellular Endocrinology 2010316 79-85. (doi:10.1016/j.mce.2009.08.009)

2 Hammes A, Andreassen TK, Spoelgen R, Raila J, Hubner N, Schulz H, Metzger J, Schweigert FJ, Luppa PB, Nykjaer A et al. Role of endocytosis in cellular uptake of sex steroids. Cell 2005122 751-762. (doi:10.1016/j. cell.2005.06.032)

3 Ding EL, Song Y, Manson JE, Hunter DJ, Lee CC, Rifai N, Buring JE, Gaziano JM \& Liu S. Sex hormone-binding globulin and risk of type 2 diabetes in women and men. New England Journal of Medicine $2009 \mathbf{3 6 1}$ 1152-1163. (doi:10.1056/NEJMoa0804381)

4 Bhasin S, Jasjua GK, Pencina M, D'Agostino R Sr, Coviello AD, Vasan RS $\&$ Travison TG. Sex hormone-binding globulin, but not testosterone, is associated prospectively and independently with incident metabolic syndrome in men: the framingham heart study. Diabetes Care 201134 2464-2470. (doi:10.2337/dc11-0888)

5 Bhasin S, Cunningham GR, Hayes FJ, Matsumoto AM, Snyder PJ, Swerdloff RS \& Montori VM. Testosterone therapy in men with androgen deficiency syndromes: an Endocrine Society clinical practice guideline. Journal of Clinical Endocrinology and Metabolism 201095 2536-2559. (doi:10.1210/jc.2009-2354)

6 Araujo AB, Dixon JM, Suarez EA, Murad MH, Guey LT \& Wittert GA. Clinical review: endogenous testosterone and mortality in men: a systematic review and meta-analysis. Journal of Clinical Endocrinology and Metabolism 201196 3007-3019. (doi:10.1210/jc.2011-1137)

7 Muraleedharan V, Marsh H, Kapoor D, Channer KS \& Jones TH. Testosterone deficiency is associated with increased risk of mortality and testosterone replacement improves survival in men with type 2 diabetes. European Journal of Endocrinology $201316925-733$. (doi:10.1530/EJE-13-0321)

8 Dhindsa S, Prabhakar S, Sethi M, Bandyopadhyay A, Chaudhuri A \& Dandona P. Frequent occurrence of hypogonadotropic hypogonadism in type 2 diabetes. Journal of Clinical Endocrinology and Metabolism 2004 89 5462-5468. (doi:10.1210/jc.2004-0804)

9 Grossmann M, Thomas MC, Panagiotopoulos S, Sharpe K, Macisaac RJ, Clarke S, Zajac JD \& Jerums G. Low testosterone levels are common and associated with insulin resistance in men with diabetes. Journal of Clinical Endocrinology and Metabolism 200893 1834-1840. (doi:10.1210/ jc.2007-2177)

10 Sikaris K, McLachlan RI, Kazlauskas R, de Kretser D, Holden CA \& Handelsman DJ. Reproductive hormone reference intervals for healthy fertile young men: evaluation of automated platform assays. Journal of Clinical Endocrinology and Metabolism 200590 5928-5936. (doi:10.1210/ jc.2005-0962)

11 Vermeulen A, Verdonck L \& Kaufman JM. A critical evaluation of simple methods for the estimation of free testosterone in serum. Journal of Clinical Endocrinology and Metabolism 199984 3666-3672. (doi:10.1210/jcem.84.10.6079)

12 R Core Team. R: A language and environment for statistical computing. R Foundation for Statistical Computing,Vienna, Austria. 2015 http://wwwR-projectorg/.

13 Fellows I. Deducer: a data analysis GUI for R. Journal of Statistical Software 201249 1-15. (doi:10.18637/jss.v049.i08)

14 Henning PC, Scofield DE, Spiering BA, Staab JS, Matheny RW Jr, Smith MA, Bhasin S \& Nindl BC. Recovery of endocrine and inflammatory mediators following an extended energy deficit. Journal of Clinical Endocrinology and Metabolism 201499 956-964.

15 Kalyani RR, Gavini S \& Dobs AS. Male hypogonadism in systemic disease. Endocrinology and Metabolism Clinics of North America 200736 333-348. (doi:10.1016/j.ecl.2007.03.014)

16 Damassa DA, Lin TM, Sonnenschein C \& Soto AM. Biological effects of sex hormone-binding globulin on androgen-induced proliferation and androgen metabolism in LNCaP prostate cells. Endocrinology 1991129 75-84. (doi:10.1210/endo-129-1-75)

17 Hryb DJ, Khan MS, Romas NA \& Rosner W. The control of the interaction of sex hormone-binding globulin with its receptor by steroid hormones. Journal of Biological Chemistry 1990265 6048-6054.

18 Kalme T, Seppala M, Qiao Q, Koistinen R, Nissinen A, Harrela M, Loukovaara M, Leinonen P \& Tuomilehto J. Sex hormone-binding globulin and insulin-like growth factor-binding protein-1 as indicators of metabolic syndrome, cardiovascular risk, and mortality in elderly men. Journal of Clinical Endocrinology and Metabolism 200590 1550-1556. (doi:10.1210/jc.2004-0762)

19 Goodman-Gruen D \& Barrett-Connor E. A prospective study of sex hormone-binding globulin and fatal cardiovascular disease in Rancho Bernardo men and women. Journal of Clinical Endocrinology and Metabolism 199681 2999-3003.

20 Araujo AB, Kupelian V, Page ST, Handelsman DJ, Bremner WJ \& McKinlay JB. Sex steroids and all-cause and cause-specific mortality in men. Archives of Internal Medicine 2007167 1252-1260. (doi:10.1001/ archinte.167.12.1252)

21 Hyde Z, Norman PE, Flicker L, Hankey GJ, Almeida OP, McCaul KA, Chubb SA \& Yeap BB. Low free testosterone predicts mortality from cardiovascular disease but not other causes: the Health in Men Study. Journal of Clinical Endocrinology and Metabolism 201297 179-189. (doi:10.1210/jc.2011-1617)

22 Pascual-Figal DA, Tornel PL, Nicolas F, Sanchez-Mas J, Martinez MD, Gracia MR, Garrido IP, Ruiperez JA \& Valdes M. Sex hormone-binding globulin: a new marker of disease severity and prognosis in men with chronic heart failure. Revista Española de Cardiología 200962 1381-1387.

23 Svartberg J, Schirmer H, Wilsgaard T, Mathiesen EB, Njolstad I, Lochen ML \& Jorde R. Single-nucleotide polymorphism, rs1799941 in the Sex Hormone-Binding Globulin (SHBG) gene, related to both 
serum testosterone and SHBG levels and the risk of myocardial infarction, type 2 diabetes, cancer and mortality in men: the Tromso Study. Andrology 20142 212-218. (doi:10.1111/j.2047-2927.2013. 00174.x)

24 Khaw KT \& Barrett-Connor E. Lower endogenous androgens predict central adiposity in men. Annals of Epidemiology 19922 675-682. (doi:10.1016/1047-2797(92)90012-F)

25 Rosner W, Auchus RJ, Azziz R, Sluss PM \& Raff H. Position statement: utility, limitations, and pitfalls in measuring testosterone: an Endocrine Society position statement. Journal of Clinical Endocrinology and Metabolism 200792 405-413. (doi:10.1210/jc.2006-1864)

26 Gianatti EJ, Dupuis P, Hoermann R, Strauss BJ, Wentworth JM, Zajac JD $\&$ Grossmann M. Effect of testosterone treatment on glucose metabolism in men with type 2 diabetes: a randomized controlled trial. Diabetes Care 201437 2098-2107. (doi:10.2337/dc13-2845)

27 Harwood DT \& Handelsman DJ. Development and validation of a sensitive liquid chromatography-tandem mass spectrometry assay to simultaneously measure androgens and estrogens in serum without derivatization. Clinica Chimica Acta 2009409 78-84. (doi:10.1016/j. cca.2009.09.003)

28 Rosner W. Sex steroids and the free hormone hypothesis. Cell $2006 \mathbf{1 2 4}$ 455-456; author reply 456-457. (doi:10.1016/j.cell.2006.01.026)

29 Basaria S, Coviello AD, Travison TG, Storer TW, Farwell WR, Jette AM, Eder R, Tennstedt S, Ulloor J, Zhang A et al. Adverse events associated with testosterone administration. New England Journal of Medicine 2010 363 109-122. (doi:10.1056/NEJMoa1000485)

Received 6 July 2015

Revised version received 8 October 2015

Accepted 19 October 2015 